\title{
Perfeccionismo, Burnout e as Atividades Extracurriculares nos Estudantes de Medicina da Universidade de Coimbra
}

\author{
Perfectionism, Burnout and Extracurricular Activities \\ Among Medical Students from the University of Coimbra
}

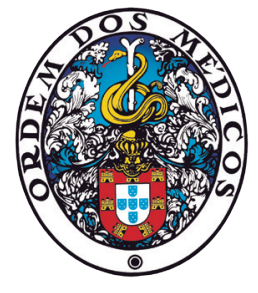

Tomás DINIS $\mathbb{1}^{1}$, Luiz Miguel SANTIAGO², Inês Rosendo CAETANO², João MARÔCO

Acta Med Port 2020 Jun;33(6):367-375 - https://doi.org/10.20344/amp.12083

RESUMO

Introdução: A participação em atividades extracurriculares reduz a importância atribuída a alguns motivos para o perfeccionismo que influenciam a prevalência de burnout nos estudantes de medicina. Este estudo visou investigar de que forma esta relação se processa e de que fatores depende, de forma a pensar estratégias direcionadas a estes potenciais alvos de atuação.

Material e Métodos: Elaborou-se um questionário capaz de avaliar a prática de atividades extracurriculares que incluiu um inquérito dos motivos para o perfeccionismo e parte do Copenhagen Burnout Inventory adapted for students. À sua aplicação, via online, aos estudantes do Mestrado Integrado em Medicina da Faculdade de Medicina da Universidade de Coimbra, seguiu-se a análise estatística e inferencial dos dados obtidos.

Resultados: Numa amostra de 360 alunos, $40 \%$ pontuaram elevados níveis de burnout pessoal e $28,1 \%$ de burnout relacionado com os estudos, tendo $22,2 \%$ obtido elevados níveis nas duas componentes. Alunos que praticavam atividades extracurriculares estiveram menos frequentemente associados a altos valores de burnout pessoal e relacionado com os estudos, simultaneamente $(p=0,013)$. 0 tipo de atividade extracurricular escolhido não mostrou relação com a prevalência de burnout.

Discussão: Apenas 53,9\% dos alunos participam numa atividade extracurricular, fator associado a uma menor prevalência de burnout, especialmente nos alunos que a realizam várias vezes por semana. A escolha de um tipo de atividade em detrimento de outro não parece influenciar a prevalência desta síndrome, estando todos associados a benefícios em diferentes domínios.

Conclusão: Verificou-se menor prevalência de burnout em estudantes que praticavam atividades extracurriculares. Será necessária a realização de estudos que nesta relação permitam inferir causalidade, de forma a formular soluções efetivas e específicas do contexto do curso médico.

Palavras-chave: Atividades de Lazer; Esgotamento Profissional; Estudantes de Medicina; Inquéritos e Questionários; Perfeccionismo; Portugal; Stress Psicológico

\section{ABSTRACT}

Introduction: The participation in extracurricular activities reduces the attributed importance to some causes of perfectionism, which influences the prevalence of burnout in medical students. This study aimed to investigate how this relationship occurs and which factors it is dependent on, in order to create strategies directed at these potential targets.

Material and Methods: We developed a questionnaire that assesses the participation in extracurricular activities, including a survey regarding the reasons for perfectionism and part of the Copenhagen Burnout Inventory adapted for students. The questionnaire's application to medical students of the Masters Degree in Medicine of the Faculty of Medicine, University of Coimbra was followed by statistical analysis.

Results: In a sample of 360 students, $40 \%$ ranked highly in personal burnout, and $28.1 \%$ in study-related burnout, while $22.2 \%$ showed high levels of burnout in both components. Students who participated in extracurricular activities were less frequently associated with simultaneous personal-related and study-related high levels of burnout $(p=0.013)$. The kind of chosen extracurricular activity was not associated with the prevalence of burnout.

Discussion: Only $53.9 \%$ of students participate in extracurricular activities. Such participation is associated with a lower prevalence of burnout if it is practiced several times per week. The choice of one kind of activity over another does not seem to influence the prevalence of this syndrome, all of which are associated with benefits in different domains.

Conclusion: We observed a lower prevalence of burnout among students that engaged in extracurricular activities. Further studies that can infer causality in this relationship are needed, in order to establish effective solutions specific to the medical course context. Keywords: Burnout, Professional; Leisure Activities; Perfectionism; Portugal; Students, Medical; Stress, Psychological; Surveys and Questionnaires

\section{INTRODUÇÃO}

A profissão médica requer dos seus profissionais certas características intelectuais e pessoais, essenciais a uma eficaz gestão dos aspetos biopsicossociais da doença e desempenho clínico. ${ }^{1,2}$ Adicionalmente, a escassa margem para o erro e a permanente incerteza ao lidar com questões de extrema sensibilidade, mostram-se uma importante fonte de stress para os estudantes de medicina, que parecem

sofrer por antecipação. ${ }^{3}$

A exposição prolongada a altos níveis de ansiedade, particularmente inerentes ao ambiente educativo associado ao curso de medicina, mostrou-se associada a uma diminuição da qualidade de vida ${ }^{4-6}$ destes estudantes, culminando frequentemente em depressão, abuso de substâncias e ideação suicida. ${ }^{3,5,7-10}$ Estes poderão vir a desenvolver

1. Faculdade de Medicina. Universidade de Coimbra. Coimbra. Portugal.

2. Clínica Universitária de Medicina Geral e Familiar. Faculdade de Medicina. Universidade de Coimbra. Coimbra. Portugal.

3. Departamento de Ciências Psicológicas. Instituto Universitário de Ciências Psicológicas, Sociais e da Vida. Lisboa. Portugal.

$\square$ Autor correspondente: Tomás Dinis. tomasdinis24@gmail.com

Recebido: 26 de março de 2019 - Aceite: 21 de outubro de 2019 | Copyright @ Ordem dos Médicos 2020 
síndrome de burnout, 4,7,8,10,11 definido por Schaufeli e Greenglass ${ }^{12}$ como "um estado de exaustão física, emocional e mental resultante de um envolvimento a longo prazo em situações de trabalho emocionalmente exigentes", que se associará a um prejuízo na prestação de cuidados e pro-

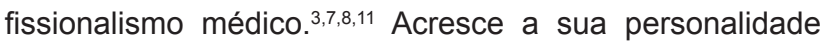
tipicamente perfeccionista, ${ }^{7,13,14}$ caracterizada pela necessidade de se ser perfeito, estabelecendo altos padrões de performance com uma forte atitude autocrítica, ${ }^{13-16}$ associada a uma maior vulnerabilidade ao burnout ${ }^{13,14}$ e ansiedade social. ${ }^{15,16}$

Mecanismos de coping, assentes numa equilibrada gestão de tempo, mostraram ter impacto na prevenção de tais consequências, 4,5,8,10,11 destacando-se as atividades extracurriculares $(A E)$, estratégias que permitem aos estudantes desenvolver capacidades interpessoais e evidenciar qualidades cruciais para o sucesso académico, especificamente na área da saúde, como atitude crítica, potencial de liderança, desejo de crescimento pessoal e consciência social. . $^{2,45,10,17}$

A vontade de contribuir para uma melhor atuação da Faculdade de Medicina da Universidade de Coimbra (FMUC) na redução da incidência de stress e burnout nos seus estudantes levou à investigação de estratégias promotoras da qualidade de vida e saúde, sendo a inclusão de AE no currículo do curso um exemplo. Por isso, e estabelecido o facto de que a inserção nestas ocupações influencia, no sentido da redução, a importância atribuída a alguns dos motivos para o perfeccionismo, ${ }^{3}$ de que depende a vulnerabilidade ao stress $^{3,6}$ e a prevalência de burnout nos alunos de medicina, 4,10,11,17 importa estudar de que forma esta relação se processa e de que fatores depende.

Foi assim objetivo deste trabalho investigar, entre os alunos do Mestrado Integrado em Medicina (MIM) da FMUC, o perfeccionismo e burnout, e a sua relação com o tipo de atividades extracurriculares praticada, regularidade e tempo de prática, explorando a sua dependência de variáveis sociodemográficas.

\section{MATERIAL E MÉTODOS}

Realizou-se um estudo observacional de uma amostra de conveniência dos estudantes de medicina do MIM-FMUC, do ano letivo de 2018/2019, através da construção de um questionário.

Este questionário incluiu um inquérito epidemiológico seguido de um inquérito acerca dos motivos para o perfeccionismo e intolerância à frustração, aplicado no âmbito de um trabalho anterior ${ }^{3}$ que convidava o aluno a atribuir, através de uma escala do tipo Likert, um grau de importância a diversos fatores - de um mínimo de (1), "Nada importante", a um máximo de (4), "Muito importante" -, avaliando o modo como eles influenciam o seu perfeccionismo e intolerância à frustração.

A terceira parte consistiu num inquérito que avaliava a prática de $\mathrm{AE}$, atividades que não contribuem diretamente para as classificações do curso, requerendo um certo nível de habilidade, dedicação ou autodisciplina. ${ }^{5}$ Caso o estu- dante estivesse, aquando do preenchimento do questionário, a praticar pelo menos uma $\mathrm{AE}$, era convidado a indicar o(s) seu(s) tipo(s), frequência e tempo de prática. Caso não praticasse, era inquirido sobre se alguma vez o teria feito e, em caso afirmativo, qual o tipo, frequência, tempo de prática e há quanto tempo o interrompera.

Por último, utilizou-se uma tradução adaptada e validada para a população portuguesa ${ }^{18}$ de um instrumento de avaliação de burnout em estudantes, o Copenhagen Burnout Inventory adapted for students (CBI-S) proposto originalmente por Kristensen et al (2005). Este instrumento é composto por diversas escalas que podem ser usadas independentemente e que avaliam o estado de fadiga/ exaustão da população em estudo, característica nuclear, por definição, da síndrome de burnout. ${ }^{12}$ Por ser objetivo do presente trabalho compreender a influência das $A E$, especificamente nas características pessoais dos estudantes da FMUC e nos seus estudos, optou-se por utilizar apenas as escalas "Burnout pessoal" (seis questões) e "Burnout relacionado com os estudos" (sete questões). Estas avaliam, respetivamente, o grau de exaustão física e psicológica experienciado pelo aluno e aquele percebido pelo mesmo como relacionado com os seus estudos, tendo igualmente mostrado maior correlação, tanto entre si, como com outros instrumentos de avaliação de burnout. ${ }^{12,18,19}$ Excluíram-se as escalas "Burnout relacionado com os colegas" e "Burnout relacionado com os professores”, já que a relação com estes não mostra ser uma manifestação importante de Burnout, ${ }^{19}$ evitando a utilização de um questionário demasiado extenso, para reduzir a probabilidade de desistência.

As questões foram avaliadas segundo uma escala do tipo Likert, com cinco opções: "Nunca" (1), "Raramente" (2), "Por vezes" (3), "Frequentemente" (4) e "Sempre" (5), posteriormente pontuadas com 0, 25, 50, 75 e 100 pontos respetivamente (exceto uma das questões, com pontuação inversa), sendo a pontuação de cada escala calculada pela média das suas respostas. ${ }^{20}$ Utilizou-se uma classificação em níveis de burnout (baixo, moderado e alto), tendo como valores de cut-off os percentis 33 e 66 , tal como acontece noutros instrumentos. ${ }^{21}$ Considerou-se que um indivíduo tinha, com maior probabilidade, síndrome de burnout, relativamente ao seu grupo, quando pontuava acima do percentil 66 simultaneamente para as duas escalas. Através da comparação entre ambas, foi possível identificar a percentagem de alunos que se encontravam exaustos, atribuindo esse estado a fatores não relacionados com os estudos. ${ }^{12}$ Para ambas as escalas do CBI-S foi verificada a consistência interna através do $\alpha$ de Cronbach, considerando-se adequada quando $\alpha>0,700 .{ }^{22}$

O questionário foi testado numa pequena amostra da população em estudo, recorrendo-se a um conjunto de alunos do MIM da Universidade da Beira Interior, respeitando a proporção entre sexos observada nos alunos da FMUC. A estes respondentes pediu-se que se manifestassem quanto à sua clareza e organização, de modo a excluir questões ambíguas ou de difícil interpretação.

A sua aplicação foi feita através da plataforma online 
da Google Forms, iniciando a fase de estudo quantitativo. A participação foi anónima, confidencial e voluntária, facto garantido pela aceitação do consentimento informado requerido para preenchimento do questionário, garantindo-se também a não duplicação de respostas. Para a sua divulgação, foram utilizados os grupos da rede social Facebook dos seis anos do MIM, mantendo o questionário disponível entre 10 de dezembro de 2018 e 10 de janeiro de 2019.

O cálculo do tamanho da amostra necessário foi feito utilizando um intervalo de confiança de 95\% e uma margem de erro de $5 \%$, tendo em conta os 1798 alunos matriculados no MIM no ano letivo de 2018/2019.

Após registo dos dados em Microsoft Excel, a análise estatística descritiva e inferencial fez-se com recurso ao programa Statistical Package for the Social Sciences SPSS. No que diz respeito à análise descritiva, as variáveis quantitativas foram caracterizadas pela sua média e desvio padrão e verificada a normalidade da sua distribuição pelo teste de Kolmogorov-Smirnov, enquanto que, para as variáveis qualitativas, se recorreu à frequência absoluta e relativa. Já a análise inferencial, para variáveis nominais, foi feita através de teste qui-quadrado $\left(\chi^{2}\right)$ e, para variáveis ordinais, com distribuição não normal, testes não paramétricos $U$ de Mann-Whitney e Kruskal-Wallis, sendo definido como estatisticamente significativo um valor de $p<0,05$.

O presente estudo obteve o parecer favorável da Comissão de Ética da FMUC.

\section{RESULTADOS}

\section{Caracterização da amostra}

Foi estudada uma amostra de conveniência de 360 alunos (sendo o tamanho de amostra necessário calculado de $\mathrm{n}=317)$, com idades compreendidas entre 17 e 36 anos (21,07 $\pm 2,68), 277$ do sexo feminino $(76,9 \%)$ e 83 do sexo masculino (23,1\%). Verificou-se uma participação de $22,2 \%$ dos alunos do primeiro ano, $18,1 \%$ do segundo ano, $11,9 \%$ do terceiro ano, $13,6 \%$ do quarto ano, $12,2 \%$ do quinto ano e $21,9 \%$ do sexto ano, residindo $71,3 \%$ em habitação não própria. No que diz respeito à "Prática de atividades extracurriculares", 53,9\% dos alunos referiu fazê-lo atualmente, facto que não revelou relacionar-se significativamente com nenhuma variável epidemiológica analisada (Tabela 1).

A distribuição da prática de AE no MIM (Tabela 2) mostrou "Desporto" e "Atividade cultural" como as AE mais escolhidas pelos alunos, em $60,3 \%$ e $35,6 \%$ dos casos, respetivamente. Apenas a escolha de "Desporto" como "Tipo de AE praticada" mostrou variação estatisticamente relevante com o ano do MIM frequentado $(p=0,036)$, sendo referida por $79,5 \%$ dos alunos do sexto ano.

A frequência de prática $(p=0,001)$ apresentou $44,4 \%$ de prática "Várias vezes por semana" pelos alunos do $1^{\circ}$ ano, enquanto que no sexto ano, $72,7 \%$ o fazia com essa frequência. Quanto ao tempo de prática $(p=0,003)$, verificou-se duração superior a três anos em mais de metade dos alunos dos primeiros e sexto anos enquanto que nos terceiros e quarto anos, apenas cerca de $30 \%$ o fez durante esse período. Por fim, para os que já tinham praticado uma $\mathrm{AE}$ no passado, o tempo que decorreu desde a sua interrupção mostrou variar com o ano de frequência $(p=$ 0,005 ) sendo que, no primeiro ano, metade seriam os que há menos de seis meses teriam interrompido a $\mathrm{AE}$, enquanto que no sexto ano, maior percentagem o fez há mais de três anos.

Tabela 1 - Caracterização da amostra estudada segundo a prática de atividades extracurriculares entre os alunos do Mestrado Integrado em Medicina da Faculdade de Medicina da Universidade de Coimbra do ano letivo de 2018/2019

\begin{tabular}{|c|c|c|c|c|c|}
\hline & & Prática de & ividades ext & curriculares & Tatal \\
\hline Variáveis & & Atual & Anterior & Nunca & \\
\hline & Masculino & $51(61.4)$ & $24(289)$ & $8(96)$ & $83(231)$ \\
\hline & Feminino & $143(51,6)$ & $107(38,6)$ & $27(9,7)$ & $277(76,9)$ \\
\hline & $1^{\circ}$ ano & $36(45,0)$ & $38(47,5)$ & $6(7,5)$ & $80(22,2)$ \\
\hline & $2^{\circ}$ ano & $29(44,6)$ & $28(43,1)$ & $8(12,3)$ & $65(18,1)$ \\
\hline Ano de & $3^{\circ}$ ano & $29(67,4)$ & $12(27,9)$ & $2(4,7)$ & $43(11,9)$ \\
\hline MIM $^{* *}$ & $4^{\circ}$ ano & $33(67,3)$ & $10(20,4)$ & $6(12,2)$ & $49(13,6)$ \\
\hline & $5^{\circ}$ ano & $23(52,3)$ & $16(36,4)$ & $5(11,4)$ & $44(12,2)$ \\
\hline & $6^{\circ}$ ano & $44(55,7)$ & $27(34,2)$ & $8(10,1)$ & $79(21,9)$ \\
\hline & Habitação própria com o agregado familiar & $51(56,7)$ & $31(34,4)$ & $8(8,9)$ & $90(25,0)$ \\
\hline & Habitação própria não partilhada & $9(69,2)$ & $4(30,8)$ & $0(0,0)$ & $13(3,6)$ \\
\hline durante $o$ & Habitação arrendada não partilhada & $11(50,0)$ & $8(36,4)$ & $3(13,6)$ & $22(6,1)$ \\
\hline periodo letivo & Quarto arrendado em habitação partilhada & $109(51,9)$ & $79(37,6)$ & $22(10,5)$ & $210(58,3)$ \\
\hline & Residência universitária & $14(56,0)$ & $9(36,0)$ & $2(8,0)$ & $25(6,9)$ \\
\hline Total & & $194(53,9)$ & $131(36,4)$ & $35(9,7)$ & $360(100,0)$ \\
\hline Idade ${ }^{* * * *}$ (média & esvio padrão) & $21,11 \pm 2,46$ & $21,02 \pm 3,1$ & $21,03 \pm 2,22$ & $21,07 \pm 2,68$ \\
\hline
\end{tabular}

*: $p=0,248 ;{ }^{* *}: p=0,103 ;{ }^{* * *}: p=0,807 ;{ }^{* * * *}: p=0,560$ 
Tabela 2 - Prática de atividades extracurriculares nos estudantes do Mestrado Integrado em Medicina da Faculdade de Medicina da Universidade de Coimbra

\begin{tabular}{|c|c|c|c|c|c|c|c|}
\hline \multirow{3}{*}{ Variáveis } & \multicolumn{6}{|c|}{ Ano de frequência no MIM } & \multirow{3}{*}{$\begin{array}{l}\text { Total } \\
\text { n (\%) }\end{array}$} \\
\hline & $1^{\circ}$ ano & $2^{\circ}$ ano & $3^{\circ}$ ano & $4^{\circ}$ ano & $5^{\circ}$ ano & $6^{\circ}$ ano & \\
\hline & $\mathrm{n}(\%)$ & $\mathrm{n}(\%)$ & n (\%) & $\mathrm{n}(\%)$ & $\mathrm{n}(\%)$ & n (\%) & \\
\hline \multicolumn{8}{|l|}{ Tipo de AE praticada } \\
\hline Desporto* & $20(55,6)$ & $12(41,4)$ & $18(62,1)$ & $18(54,5)$ & $14(60,9)$ & $35(79,5)$ & $117(60,3)$ \\
\hline Atividade Cultural & $11(30,6)$ & $16(55,2)$ & $12(41,4)$ & $12(36,4)$ & $9(39,1)$ & $9(20,5)$ & $69(35,6)$ \\
\hline Associativismo & $8(22,2)$ & $6(20,7)$ & $3(10,3)$ & $6(18,2)$ & $8(34,8)$ & $9(20,5)$ & $40(20,6)$ \\
\hline Serviço Social & $4(11,1)$ & $7(24,1)$ & $3(10,3)$ & $5(15,2)$ & $4(17,4)$ & $7(15,9)$ & $30(15,5)$ \\
\hline Atividade Remunerada & $1(2,8)$ & $0(0,0)$ & $2(6,9)$ & $3(9,1)$ & $3(13,0)$ & $1(2,3)$ & $10(5,2)$ \\
\hline \multicolumn{8}{|l|}{ Frequência de prática** } \\
\hline Todos os dias & $1(2,8)$ & $3(10,3)$ & $1(3,4)$ & $2(6,1)$ & $5(21,7)$ & $5(11,4)$ & $17(8,8)$ \\
\hline Várias vezes por semana & $16(44,4)$ & $11(37,9)$ & $22(75,9)$ & $23(69,7)$ & $13(56,5)$ & $32(72,7)$ & $117(60,3)$ \\
\hline Uma vez por semana & $13(36,1)$ & $9(31,0)$ & $6(20,7)$ & $6(18,2)$ & $4(17,4)$ & $6(13,6)$ & $44(22,7)$ \\
\hline 1 - 3 vezes por mês & $4(11,1)$ & $3(10,3)$ & $0(0,0)$ & $2(6,1)$ & $0(0,0)$ & $1(2,3)$ & $10(5,2)$ \\
\hline Ocasionalmente & $2(5,6)$ & $3(10,3)$ & $0(0,0)$ & $0(0,0)$ & $1(4,3)$ & $0(0,0)$ & $6(3,1)$ \\
\hline \multicolumn{8}{|l|}{ Tempo de prática*** } \\
\hline Menos de 6 meses & $8(10,8)$ & $6(10,5)$ & $6(14,6)$ & $8(18,6)$ & $2(5,1)$ & $0(0,0)$ & $30(9,2)$ \\
\hline 6 meses a 1 ano & $8(10,8)$ & $10(17,5)$ & $9(22,0)$ & $6(14,0)$ & $7(17,9)$ & $7(9,9)$ & $47(14,5)$ \\
\hline 1 a 2 anos & $10(13,5)$ & $8(14,0)$ & $10(24,4)$ & $10(23,3)$ & $7(17,9)$ & $15(21,1)$ & $60(18,5)$ \\
\hline 2 a 3 anos & $5(6,8)$ & $4(7,0)$ & $4(9,8)$ & $6(14,0)$ & $5(12,8)$ & $11(15,5)$ & $35(10,8)$ \\
\hline Mais de 3 anos & $43(58,1)$ & $29(50,9)$ & $12(29,3)$ & $13(30,2)$ & $18(46,2)$ & $38(53,5)$ & $153(47,1)$ \\
\hline \multicolumn{8}{|c|}{ Tempo de interrupção da $A E$ anterior**** } \\
\hline Há menos de 6 meses & $19(50,0)$ & $3(10,7)$ & $1(8,3)$ & $3(30,0)$ & $2(12,5)$ & $9(33,3)$ & $37(28,2)$ \\
\hline Há 6 meses a 1 ano & $5(13,2)$ & $5(17,9)$ & $2(16,7)$ & $3(30,0)$ & $3(18,8)$ & $3(11,1)$ & $21(16,0)$ \\
\hline Há 1 a 2 anos & $10(26,3)$ & $11(39,3)$ & $2(16,7)$ & $1(10,0)$ & $3(18,8)$ & $2(7,4)$ & $29(22,1)$ \\
\hline Há 2 a 3 anos & $1(2,6)$ & $5(17,9)$ & $7(58,3)$ & $1(10,0)$ & $1(6,3)$ & $2(7,4)$ & $17(13,0)$ \\
\hline Há mais de 3 anos & $3(7,9)$ & $4(14,3)$ & $0(0,0)$ & $2(20,0)$ & $7(43,8)$ & $11(40,7)$ & $27(20,6)$ \\
\hline
\end{tabular}

${ }^{*}: p=0,036 ;{ }^{* *}: p=0,001 ;{ }^{* * *}: p=0,003 ;{ }^{* * *}: p=0,005$

Tabela 3 - Níveis de burnout nos estudantes do MIM da FMUC do ano letivo 2018/2019

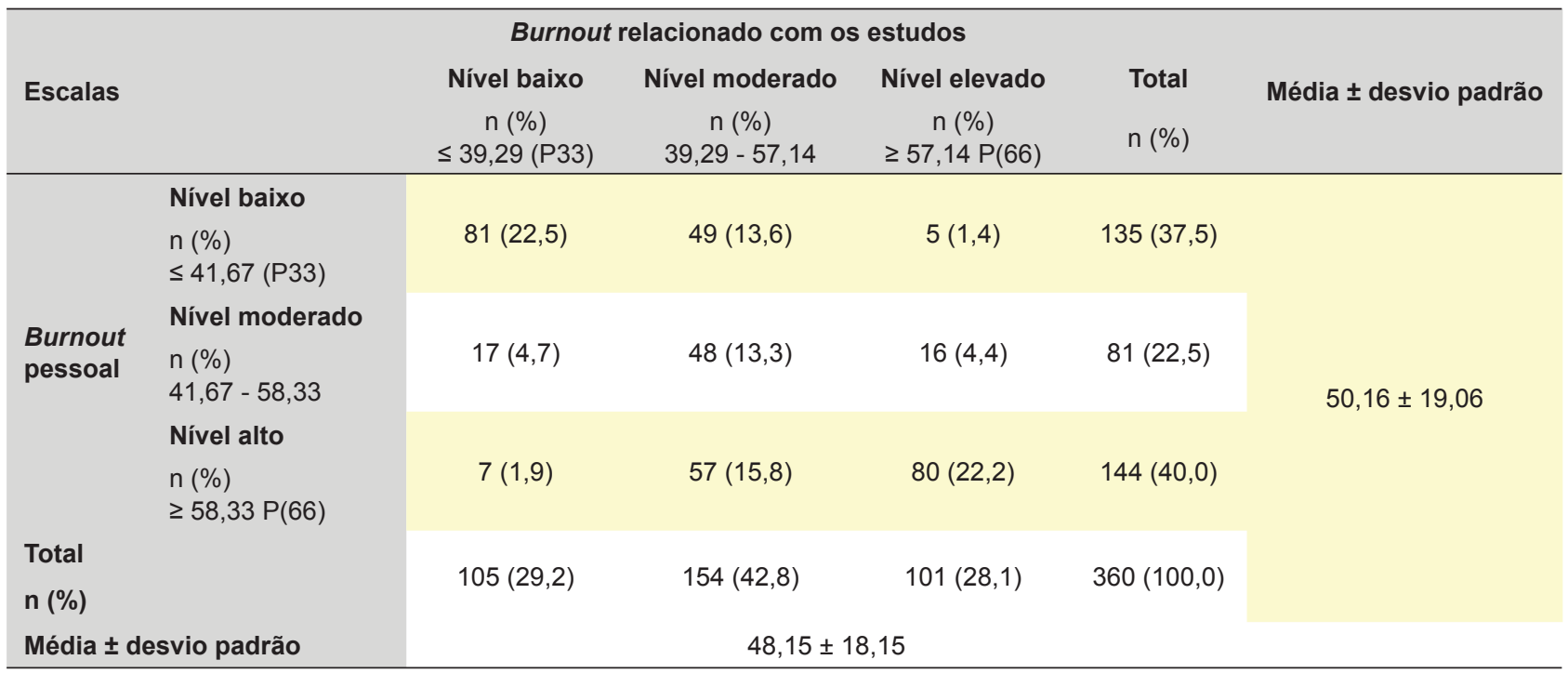

\section{Burnout}

Na Tabela 3, apresenta-se a distribuição dos níveis de burnout nos alunos do MIM, bem como as pontuações de cada escala, obtidas através da fórmula para as médias dos itens de cada dimensão das escalas do CBI-S. Da amostra estudada, $40 \%$ dos estudantes obtiveram pontuações 
compatíveis com altos níveis de burnout pessoal, e em $28,1 \%$ da amostra foram verificados altos níveis de burnout relacionado com os estudos. A parcela de alunos com elevados níveis de burnout pessoal mas baixos ou moderados níveis de burnout relacionado com os estudos foi de $17,7 \%$. Finalmente, 80 alunos (22,2\% da população) apresentaram altos níveis de burnout geral (variável que considera altos níveis de burnout pessoal e relacionado com os estudos, simultaneamente).

Estas duas escalas obtiveram um coeficiente de correlação de Spearman de 0,731 ( $p<0,001)$, indicando a existência de forte associação entre burnout pessoal e o burnout relacionado com os estudos, constructos que apresentaram bons índices de consistência interna, $\alpha=0,866$ e $\alpha=0,848$, respetivamente.

A associação das variáveis epidemiológicas com a prática de $\mathrm{AE}$ e os níveis de burnout encontra-se exposta na Tabela 4, incluindo as pontuações obtidas pelas escalas aplicadas. Para simplificação, foram omitidas a "Idade" ( $p=$ $0,052)$, a "Residência durante o período letivo" $(p=0,176)$ e o "Tempo de prática da $A E$ " ( $p=0,644)$, que não revelaram relação estatisticamente significativa.

O burnout pessoal mostrou relação com o sexo ( $p=$ 0,009 ), com $43 \%$ do sexo feminino a apresentar altos níveis desta variável. Também com o ano de frequência foi verificada relação estatisticamente significativa $(p=0,012)$. Apenas $34 \%$ dos que praticavam uma $\operatorname{AE}(p=0,013)$ apresentaram altos níveis de burnout pessoal, componente que se

Tabela 4 - Proporção de estudantes do MIM da FMUC com altos níveis de burnout, a nível geral e em cada um dos seus domínios especificamente avaliados, em função de variáveis epidemiológicas e da atividade extracurricular

\begin{tabular}{|c|c|c|c|c|c|c|c|c|}
\hline \multicolumn{3}{|c|}{ Variáveis } & \multicolumn{2}{|c|}{$\begin{array}{l}\text { Nível alto de burnout } \\
\text { pessoal }\end{array}$} & \multicolumn{2}{|c|}{$\begin{array}{l}\text { Nível alto de burnout } \\
\text { relacionado com os estudos }\end{array}$} & \multicolumn{2}{|c|}{$\begin{array}{c}\text { Nível alto de burnout } \\
\text { geral }\end{array}$} \\
\hline & & & \multirow{3}{*}{$\begin{array}{c}n(\%) \\
25(30,1) \\
119(43,0)\end{array}$} & $p$ & $\mathrm{n}(\%)$ & $p$ & $\mathrm{n}(\%)$ & $p$ \\
\hline$\stackrel{\circ}{x}$ & Masculino & & & \multirow{2}{*}{0,009} & $22(26,5)$ & \multirow{2}{*}{0,413} & $16(19,3)$ & \multirow{2}{*}{0,283} \\
\hline ळ & Feminino & & & & $79(28,5)$ & & $64(23,1)$ & \\
\hline \multirow{6}{*}{ 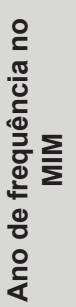 } & $1^{\circ}$ ano & & $26(32,5)$ & \multirow{6}{*}{0,012} & $14(17,5)$ & \multirow{6}{*}{0,764} & $9(11,3)$ & \multirow{6}{*}{0,563} \\
\hline & $2^{\circ}$ ano & & $38(58,5)$ & & $26(40,0)$ & & $23(35,4)$ & \\
\hline & $3^{\circ}$ ano & & $19(44,2)$ & & $15(34,9)$ & & $12(27,9)$ & \\
\hline & $4^{\circ}$ ano & & $15(30,6)$ & & $8(16,3)$ & & $7(14,3)$ & \\
\hline & $5^{\circ}$ ano & & $20(45,5)$ & & $17(38,6)$ & & $14(31,8)$ & \\
\hline & $6^{\circ}$ ano & & $26(32,9)$ & & $21(26,6)$ & & $15(19,0)$ & \\
\hline \multirow{3}{*}{ 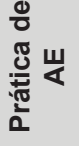 } & Atual & & $66(34,0)$ & \multirow{3}{*}{0,013} & $45(23,2)$ & \multirow{3}{*}{0,050} & $32(16,5)$ & \multirow{3}{*}{0,013} \\
\hline & Anterior & & $61(46,6)$ & & $46(35,1)$ & & $40(30,5)$ & \\
\hline & Nunca & & $17(48,6)$ & & $10(28,6)$ & & $8(22,9)$ & \\
\hline \multirow{5}{*}{ 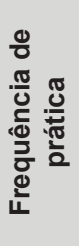 } & Todos os dias & & $4(23,5)$ & \multirow{5}{*}{0,001} & $3(17,6)$ & & $3(17,6)$ & \multirow{5}{*}{0,370} \\
\hline & Várias vezes por s & Iana & $36(30,8)$ & & $27(23,1)$ & & $17(14,5)$ & \\
\hline & Uma vez por sema & & $16(36,4)$ & & $9(20,5)$ & 0,502 & $7(15,9)$ & \\
\hline & 1 - 3 vezes por mê & & $6(60,0)$ & & $3(30,0)$ & & $3(30,0)$ & \\
\hline & Ocasionalmente & & $4(66,7)$ & & $3(50,0)$ & & $2(33,3)$ & \\
\hline \multirow{5}{*}{ 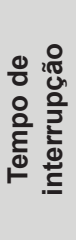 } & Há menos de 6 me & & $14(37,8)$ & \multirow{5}{*}{0,014} & $10(27,0)$ & & $9(24,3)$ & \multirow{5}{*}{0,473} \\
\hline & Há 6 meses a 1 an & & $10(47,6)$ & & $9(42,9)$ & & $7(33,3)$ & \\
\hline & Há 1 a 2 anos & & $16(55,2)$ & & $11(37,9)$ & 0,823 & $9(31,0)$ & \\
\hline & Há 2 a 3 anos & & $9(52,9)$ & & $7(41,2)$ & & $7(41,2)$ & \\
\hline & Há mais de 3 anos & & $12(44,4)$ & & $9(33,3)$ & & $8(29,6)$ & \\
\hline \multirow{10}{*}{$\begin{array}{l}\text { ய } \\
\stackrel{0}{0} \\
\circ \\
\text { 을 }\end{array}$} & \multirow{2}{*}{ Desporto } & Não & $63(45,0)$ & \multirow{2}{*}{0,239} & $41(29,3)$ & \multirow{2}{*}{0,426} & $34(24,3)$ & \multirow{2}{*}{0,266} \\
\hline & & Sim & $81(36,8)$ & & $60(27,3)$ & & $46(20,9)$ & \\
\hline & Atividade Cultural & Não & $97(42,4)$ & \multirow{2}{*}{0,247} & $64(27,9)$ & 071 & $52(22,7)$ & 0539 \\
\hline & & Sim & $47(35,9)$ & & $37(28,2)$ & , & $28(21,4)$ & ( \\
\hline & Associativismo & Não & $115(40,4)$ & 0,782 & $78(27,4)$ & 0628 & $63(22,1)$ & 0.514 \\
\hline & & Sim & $29(38,7)$ & 0,102 & $23(30,7)$ & $0,0<0$ & $17(22,7)$ & 0,014 \\
\hline & Servico Social & Não & $112(38,4)$ & & $81(27,7)$ & 0503 & $63(21,6)$ & 0321 \\
\hline & Getviģo suctâl & Sim & $32(47,1)$ & $0, \angle 02$ & $20(29,4)$ & 0,003 & $17(25,0)$ & $0,0<1$ \\
\hline & Atividade & Não & $133(39,3)$ & 0386 & $93(27,5)$ & 0516 & $75(22,2)$ & 0564 \\
\hline & Remunerada & Sim & $11(50,0)$ & 0,000 & $8(36,4)$ & (ס) & $5(22,7)$ & ז \\
\hline
\end{tabular}


viu menos prevalente na prática diária de $\operatorname{AE}(p=0,001)$. O abandono da $\operatorname{AE}(p=0,014)$ há menos de seis meses mostrou-se associado a menor percentagem de alunos com níveis elevados de burnout pessoal. O valor de burnout geral apenas apontou relação estatisticamente relevante com a prática de $\mathrm{AE}(p=0,013)$, com altos níveis de burnout mais frequentemente verificados em alunos que não se encontravam a realizar atividades extracurriculares ou que nunca o tinham feito. $O$ tipo de $A E$ escolhido não mostrou relação significativa com a prevalência de qualquer componente de burnout.

\section{Motivos para o perfeccionismo e intolerância à frustra- ção}

A importância atribuída aos motivos para o perfeccionismo e intolerância à frustração pelos alunos que não praticavam $A E$ não mostrou variar significativamente face àqueles que nelas se encontravam inseridos. Entre estes últimos, não se verificou, da mesma forma, variação com o seu tempo de realização.

Apesar disso, entre os alunos que realizavam $\mathrm{AE}$ à altura do estudo, e considerando a frequência da sua prática (Tabela 5), os "Fatores intrínsecos (do próprio)" $(p=0,048)$ mostraram ser um motivo "Muito importante" para 35,3\% dos alunos que as praticavam "Todos os dias", mas, para os que o faziam "Ocasionalmente", esse grau foi atribuído pela totalidade. A "Pressão ambiental" $(p=0,028)$, para quem praticava $\mathrm{AE}$ "Todos os dias"/"Várias vezes por semana", foi um fator "Pouco"/“Nada importante" em 35,3\% e $32,5 \%$ dos casos, respetivamente. No entanto, para quem o fazia apenas "Uma vez por semana", quase metade dos casos $(47,7 \%)$ o referiu. Relativamente à "Insegurança sobre a formação profissional" ( $p=0,030), 36,4 \%$ dos que praticavam "Uma vez por semana" disseram ser um motivo "Pouco"/“Nada importante" sendo, para todos os restantes, "Importante"/“Muito importante" em $80 \%$ dos casos

Tabela 5 - Motivos "Fatores intrínsecos (do próprio)", "Pressão ambiental", "Insegurança sobre a formação profissional" e "Exigências curriculares do MIM" em função da frequência de prática da atividade extracurricular realizada

\begin{tabular}{|c|c|c|c|c|c|c|}
\hline \multirow{2}{*}{ Variáveis } & & \multicolumn{5}{|c|}{ Frequência de prática da atividade extracurricular atual } \\
\hline & & Todos os dias & $\begin{array}{l}\text { Várias vezes } \\
\text { por semana }\end{array}$ & $\begin{array}{l}\text { Uma vez por } \\
\text { semana }\end{array}$ & $\begin{array}{c}1 \text { - } 3 \text { vezes } \\
\text { por mês }\end{array}$ & Ocasionalmente \\
\hline \multirow{4}{*}{ 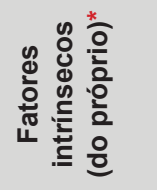 } & Nada importante & $1(5,9)$ & $1(0,9)$ & $1(2,3)$ & $0(0,0)$ & $0(0,0)$ \\
\hline & Pouco importante & $1(5,9)$ & $11(9,4)$ & $1(2,3)$ & $0(0,0)$ & $0(0,0)$ \\
\hline & Importante & $9(52,9)$ & $49(41,9)$ & $19(43,2)$ & $3(30,0)$ & $0(0,0)$ \\
\hline & Muito importante & $6(35,3)$ & $56(47,9)$ & $23(52,3)$ & $7(70,0)$ & $6(100,0)$ \\
\hline \multirow{4}{*}{ 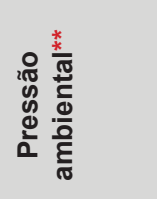 } & Nada importante & $4(23,5)$ & $4(3,4)$ & $2(4,5)$ & $0(0,0)$ & $0(0,0)$ \\
\hline & Pouco importante & $2(11,8)$ & $34(29,1)$ & $19(43,2)$ & $0(0,0)$ & $0(0,0)$ \\
\hline & Importante & $8(47,1)$ & $57(48,7)$ & $15(34,1)$ & $7(70,0)$ & $3(50,0)$ \\
\hline & Muito importante & $3(17,6)$ & $22(18,8)$ & $8(18,2)$ & $3(30,0)$ & $3(50,0)$ \\
\hline \multirow{8}{*}{ 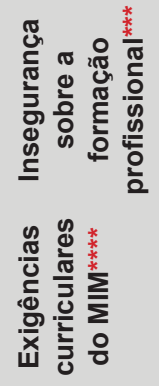 } & Nada importante & $1(5,9)$ & $5(4,3)$ & $1(2,3)$ & $1(10,0)$ & $0(0,0)$ \\
\hline & Pouco importante & $1(5,9)$ & $15(12,8)$ & $15(34,1)$ & $1(10,0)$ & $0(0,0)$ \\
\hline & Importante & $8(47,1)$ & $53(45,3)$ & $16(36,4)$ & $6(60,0)$ & $1(16,7)$ \\
\hline & Muito importante & $7(41,2)$ & $44(37,6)$ & $12(27,3)$ & $2(20,0)$ & $5(83,3)$ \\
\hline & Nada importante & $0(0,0)$ & $2(1,7)$ & $0(0,0)$ & $0(0,0)$ & $0(0,0)$ \\
\hline & Pouco importante & $4(23,5)$ & $17(14,5)$ & $2(4,5)$ & $1(10,0)$ & $1(16,7)$ \\
\hline & Importante & $6(35,3)$ & $52(44,4)$ & $13(29,5)$ & $4(40,0)$ & $1(16,7)$ \\
\hline & Muito importante & $7(41,2)$ & $46(39,3)$ & $29(65,9)$ & $5(50,0)$ & $4(66,7)$ \\
\hline
\end{tabular}

*: $p=0,048 ; * *: p=0,028 ;{ }^{* * *}: p=0,030 ; * * * *: p=0,027$

Tabela 6 - Motivo "Insegurança sobre a formação profissional" e o tempo de interrupção da atividade extracurricular previamente praticada

\begin{tabular}{|c|c|c|c|c|c|c|}
\hline \multirow{2}{*}{ Variáveis } & & \multicolumn{5}{|c|}{ Há quanto tempo se interrompeu a atividade extracurricular pregressa } \\
\hline & & Menos de 6 meses & 6 meses a 1 ano & 1 a 2 anos & 2 a 3 anos & Mais de 3 anos \\
\hline \multirow{4}{*}{ 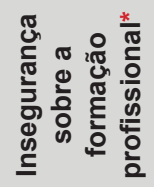 } & Nada importante & $1(2,7)$ & $0(0,0)$ & $3(10,3)$ & $0(0,0)$ & $1(3,7)$ \\
\hline & Pouco importante & $11(29,7)$ & $5(23,8)$ & $5(17,2)$ & $2(11,8)$ & $2(7,4)$ \\
\hline & Importante & $17(45,9)$ & $8(38,1)$ & $15(51,7)$ & $6(35,3)$ & $12(44,4)$ \\
\hline & Muito importante & $8(21,6)$ & $8(38,1)$ & $6(20,7)$ & $9(52,9)$ & $12(44,4)$ \\
\hline
\end{tabular}


ou mais. Por fim, as "Exigências curriculares do MIM" ( $p$ $=0,027$ ) mostraram ser um motivo "Muito importante" em pelo menos metade dos alunos que optavam pela frequência de "Uma vez por semana" ou inferior mas, nos que o faziam diariamente, apenas $41,2 \%$ o indicou.

Por fim, considerando aqueles que não praticavam nenhuma $A E$ mas que o haviam feito no passado, o tempo de interrupção da mesma mostrou relação com a importância atribuída à "Insegurança sobre a formação profissional" ( $p$ $=0,035$ ) - Tabela 6 , havendo maior atribuição de "Muito importante" nos que a abandonaram há dois ou mais anos comparando com os que o fizeram há menos de seis meses.

\section{DISCUSSÃO}

O stress prolongado a que a comunidade estudantil se encontra sujeita prejudica o seu desempenho académico, saúde mental e profissionalismo, ${ }^{3,5,6,8,11}$ motivando a realização de estudos no âmbito de determinantes da sua vulnerabilidade, de que são exemplos o perfeccionismo e o burnout. ${ }^{1,3,6,9}$

Por sua vez, as atividades extracurriculares surgem como mecanismos de coping eficazes, ${ }^{10,11}$ querendo o presente trabalho compreender e aprofundar a relação desempenhada por estas na natureza tipicamente perfeccionista do estudante de medicina, ${ }^{7,13,14}$ e na prevalência de burnout.

Os resultados revelam que, entre os estudantes de medicina da FMUC, 40\% apresenta altos níveis de burnout pessoal e $28,1 \%$ altos níveis de burnout relacionado com os estudos. As pontuações obtidas foram, em média, muito superiores (50,2 para o burnout pessoal e 48,2 para o burnout relacionado com os estudos) às observadas num estudo prospetivo levado a cabo por Kristensen et $a l,{ }^{12}$ entre profissionais de saúde e recursos humanos: 35,9 e 33, respetivamente. Além disso, 22,2\% dos estudantes pontua simultaneamente altos índices das duas componentes, vaIor que, apesar de não constituir uma pontuação geral de burnout completa, não pode deixar de ser tomada em consideração. Em 17,7\% dos alunos observam-se elevados níveis de burnout pessoal, mas não de burnout relacionado com os estudos, atribuindo assim o seu estado de exaustão a fatores não académicos como, por exemplo, problemas de saúde ou familiares, ${ }^{12}$ de que podem ser exemplos a má saúde mental $^{8}$ e a pressão exercida por familiares médicos. $^{4}$

A análise dos dados demográficos permite constatar que o sexo feminino se encontra associado a altos níveis de burnout pessoal, destacando-se também que mais de metade $(58,5 \%)$ dos alunos do segundo ano do MIM acusam valores elevados desta componente, prevalência que decresce com a progressão no curso. A confrontação destes resultados com a bibliografia é limitada pelos instrumentos de avaliação utilizados. Seria por isso interessante a realização de mais estudos com recurso à escala $\mathrm{CBI}-\mathrm{S}$ de modo a estabelecer estas comparações.

Mais de metade dos alunos do MIM-FMUC praticam uma $\operatorname{AE}(53,9 \%)$, valor que se encontra abaixo daqueles relatados noutros trabalhos internacionais. 3,4,10,17 Esta participação apresenta o maior valor nos terceiro e quarto anos (aproximadamente 67\%), sendo inferior nos restantes, distribuição presumivelmente devida à adaptação inicial ao novo ritmo de trabalho do ensino superior ${ }^{3,9,17}$ seguida da perceção da necessidade de aquisição de mecanismos que ajudem a lidar com o stress. . $^{71,17}$

Aos alunos que não se encontram a praticar $A E$ foram associados altos níveis de burnout, reforçando, e tendo em conta a bibliografia praticamente unânime, o papel protetor de tais ocupações face a este estado de exaustão. 4,5,10,11,23,24 De facto, alunos que abandonaram a sua atividade há mais tempo, não só mostram maior prevalência de altos níveis de burnout pessoal, como valorizam a insegurança sobre a formação profissional como motivo para seu o perfeccionismo. Esta observação poderá estar relacionada com o ganho de competências interpessoais e a redução de ansiedade e stress inerentes à prática de $A E, 4,10$ levando o seu abandono a maior insegurança face à capacidade de resolução de problemas, com maior dependência da experiência dada pela formação académica.

Verifica-se uma preferência pelo desporto, mencionado por $60,3 \%$ dos participantes, valor inferior quando considerada a totalidade da população, comprovando a reduzida prática desportiva entre os estudantes universitários, talvez por isso mais vulneráveis ao stress. ${ }^{23,25,26}$ Apesar disso, o burnout não varia significativamente com a atividade física, não invalidando os seus inúmeros benefícios no estilo de vida dos alunos, ${ }^{4,10,11,23-25}$ mas antes revelando que a sua escolha, em detrimento de outras $A E$, não determina maior redução na frequência de burnout.

Também às atividades culturais, como a música, se associam efeitos benéficos na redução da incidência de burnout, particularmente na comunidade médica, sendo uma estratégia, com boa razão custo-benefício, que melhora a empatia e compaixão. ${ }^{10,11} \mathrm{O}$ voluntariado (incluído em "serviços sociais") promove a solidariedade, responsabilidade social e sentido de comunidade ${ }^{10}$ qualidades fulcrais em prestadores de cuidados. Por fim, destacam-se as atividades que envolvem a organização e a liderança ("Associativismo"), que se verifica refletirem num melhor desempenho académico, ${ }^{4}$ afetando positivamente a incidência de burnout.

A frequência de prática da $A E$ aumenta com a progressão no curso, com mais alunos a optar pela realização das mesmas várias vezes por semana, aspeto relacionado com a desvalorização dos fatores intrínsecos e das exigências curriculares do MIM, como motivos para o perfeccionismo. Nestes, verifica-se também menor prevalência de burnout pessoal, achado que vem contrariar um estudo anterior que negou relação entre a frequência de prática de $\mathrm{AE}$ e o burnout. ${ }^{4} \mathrm{~A}$ prática regular e frequente de $\mathrm{AE}$ parece então ajudar a lidar com o perfeccionismo auto-orientado, dimensão nuclear deste traço de personalidade que se encontra geralmente elevada entre os estudantes de medicina, ${ }^{13}$ além do já provado benefício na performance académica ${ }^{25} \mathrm{e}$, por conseguinte, na qualidade de vida. ${ }^{5}$ 
A duração da prática de $\mathrm{AE}$, mais prolongada nos primeiro e sexto anos, não influencia nem a prevalência de burnout nem os motivos para o perfeccionismo. No entanto, um estudo longitudinal abrangente realizado, verificou que um envolvimento em $\mathrm{AE}$ mais prolongado se tornava preditor de maior resiliência psicológica e aproveitamento escolar dos adolescentes. ${ }^{27}$

Este estudo fez recurso a um questionário aplicado online método que o tornou limitado ao voluntarismo. Há também que considerar o viés de aceitabilidade social associado à utilização de escalas do tipo Likert, numa população tipicamente perfeccionista. ${ }^{7,13,14}$ Para evitar a elaboração de um constructo demasiado extenso optou-se pela não utilização de todas as escalas que integram o $\mathrm{CBI}-\mathrm{S}$, o que, apesar de possível, ${ }^{12}$ poderá gerar limitações na interpretação do valor global de burnout geral calculado. Além disso, a comparação dos resultados obtidos com a bibliografia é limitada pela escassa utilização desta escala. O estudo, apesar de analisar uma amostra de tamanho representativo, respeitando a proporção de sexos característica da população do curso de medicina da $\mathrm{FMUC}^{3}$, foi limitado a uma faculdade, sendo de caráter transversal. Tal invalida tanto a atribuição de causalidade, como a extrapolação de conclusões para a comunidade estudantil médica portuguesa. Torna-se importante a realização de estudos com o mesmo âmbito noutras faculdades do país e nesta, seguindo a coorte dos agora estudados.

Apesar destas limitações, é possível formular sugestões que possibilitem a melhoria da qualidade de vida dos estudantes de medicina da FMUC. É do interesse das escolas médicas o estabelecimento de um ambiente de ensino favorecedor da formação de profissionais que reúnam competências fulcrais ao bom desempenho dos cuidados médicos. ${ }^{4,11}$ Por todas as vantagens associadas às atividades extracurriculares, considera-se crucial o incentivo formal à sua prática, potenciando os fatores que a motivam e eliminando os seus entraves. ${ }^{10,11,17,23}$ Sugere-se assim: a criação de programas de tutoria que guiem os estudantes no ingresso controlado nas $A E$, sem prejudicar o seu desempenho académico ${ }^{4,6,10,14,17}$ e a valorização de um currículo plurivalente no ingresso na carreira médica, não apenas baseado na classificação final da Prova Nacional de Acesso, um dos principais motivos para a realização destas atividades em países que o tomam em consideração. ${ }^{17}$

Tendo em conta a elevada prevalência de burnout, é também necessária uma maior atenção à saúde mental dos estudantes por parte dos profissionais envolvidos no ensino $4,10,11$ por meio de medidas como a promoção de programas de apoio psicossocial, ${ }^{3,6,8}$ a educação para estratégias organizacionais ${ }^{5,8,14}$ e a partilha de experiências por parte de médicos séniores, focando a incerteza inerente aos atos da profissão numa perspetiva humanista da medicina. ${ }^{3,11}$

\section{CONCLUSÃO}

A prevalência de burnout nos estudantes de medicina da FMUC, estimada na sua dimensão pessoal e naquela relacionada com os estudos, mostrou ser considerável, não devendo por isso ser negligenciada, dadas as potenciais implicações no desempenho profissional futuro.

As atividades extracurriculares surgem como mecanismos de coping eficazes na gestão do stress a que os estudantes se encontram sujeitos, verificando-se que pouco mais de metade dos alunos recorrem às mesmas. Nestes, observa-se uma menor prevalência de altos valores de burnout, especialmente naqueles que o fazem várias vezes por semana, que acabam desvalorizando os fatores intrínsecos como motivadores do seu perfeccionismo.

Os resultados encontrados não permitem apontar um tipo de AE mais benéfico, em detrimento de outro, nem a duração ideal para a sua prática. Constatou-se, no entanto, uma associação positiva entre a prevalência de burnout e o abandono mais prolongado destas atividades. Tais factos deverão ser encorajadores da adoção de qualquer tipo de AE em qualquer que seja a fase do percurso académico em que o aluno já se encontre.

Apesar das importantes relações encontradas, será necessária a realização de outros estudos que demonstrem se, de facto, a menor prevalência de burnout decorre efetivamente da prática das $\mathrm{AE}$ e, confirmando-se, por que mecanismo este processo ocorre. Desta forma, será possível a criação e implementação de medidas específicas do contexto do curso médico, visando o objetivo último da melhoria da qualidade de vida dos seus estudantes.

\section{PROTEÇÃO DE PESSOAS E ANIMAIS}

Os autores declaram que os procedimentos seguidos estavam de acordo com os regulamentos estabelecidos pelos responsáveis da Comissão de Investigação Clínica e Ética e de acordo com a Declaração de Helsínquia da Associação Médica Mundial.

\section{CONFIDENCIALIDADE DOS DADOS}

Os autores declaram ter seguido os protocolos do seu centro de trabalho acerca da publicação de dados.

\section{CONFLITOS DE INTERESSE}

Os autores declaram não terem qualquer conflito de interesse relativamente ao presente artigo.

\section{FONTES DE FINANCIAMENTO}

Os autores declaram não ter recebido subsídios ou bolsas para a elaboração do artigo.

\section{REFERÊNCIAS}

1. Marques M, Macedo A, Soares M, Maia B, Pereira A, Bos S, et al. O premedical syndrome: será que existe em Portugal? Acta Med Port. 2009;22:789-96.

2. Kim S. Extracurricular activities of medical school applicants. Korean J
Med Educ. 2016;28:201-7.

3. Batista S, Santiago L, Rosendo I. Motivos para o perfeccionismo e intolerância à frustração nos estudantes de medicina da universidade de coimbra. Acta Med Port. 2008;31:527-33. 
4. Almalki S, Almojali A, Alothman A, Masuadi E, Alaqeel M. Burnout and its association with extracurricular activities among medical students in Saudi Arabia. Int J Med Educ. 2017;8:144-50.

5. Lumley S, Ward P, Roberts L, Mann J. Self-reported extracurricular activity, academic success, and quality of life in UK medical students. Int J Med Educ. 2015;6:111-7.

6. Pereira A, Santiago L, Simões J. Qualidade de vida e vulnerabilidade ao stress nos estudantes de medicina dos $5^{\circ}$ e $6^{\circ}$ anos. Coimbra: Faculdade de Medicina da Unviersidade de Coimbra; 2017.

7. Moir F, Yielder J, Sanson J, Chen Y. Depression in medical students: current insights. Adv Med Educ Pract. 2018;9:323-33.

8. Chunming W, Harrison R, Maclntyre R, Travaglia J, Balasooriya C. Burnout in medical students: a systematic review of experiences in Chinese medical schools. BMC Med Educ. 2017;17:1-11.

9. Gómez H, Pérez C, Parra P, Ortiz L, Matus O, McColl P, et al. Relación entre el bienestar y el rendimiento académico en alumnos de primer año de medicina. Rev Med Chil. 2015;143:930-7.

10. Fares J, Saadeddin Z, Al Tabosh H, Aridi H, El Mouhayyar C, Koleilat $M$, et al. Extracurricular activities associated with stress and burnout in preclinical medical students. J Epidemiol Glob Health. 2016;6:177-85.

11. Fares J, Al Tabosh H, Saadeddin Z, El Mouhayyar C, Aridi H. Stress, burnout and coping strategies in preclinical medical students. $\mathrm{N} \mathrm{Am} \mathrm{J}$ Med Sci. 2016;8:75-81.

12. Kristensen T, Borritz M, Villadsen E, Christensen K. The Copenhagen Burnout Inventory: a new tool for the assessment of burnout. Work Stress. 2005;19:192-207.

13. Seeliger $\mathrm{H}$, Harendza S. Is perfect good? - Dimensions of perfectionism in newly admitted medical students. BMC Med Educ. 2017;17:1-7.

14. Yu J, Chae S, Chang $\mathrm{K}$. The relationship among self-efficacy, perfectionism and academic burnout in medical school students. Korean J Med Educ. 2016;28:49-55.

15. Mohammadian $Y$, Mahaki B, Dehghani M, Vahid M, Lavasani F. Investigating the role of interpersonal sensitivity, anger, and perfectionism in social anxiety. Int J Prev Med. 2018;9:2.

16. Besharat $M$, Issazadegan $A$, Etemadinia $M$, Golssanamlou $S$,
Abdolmanafi A. Risk factors associated with depressive symptoms among undergraduate students. Asian J Psychiatr. 2014;10:21-6.

17. Almasry M, Kayali Z, Alsaad R, Alhayaza G, Ahmad M, Obeidat A, et al. Perceptions of preclinical medical students towards extracurricular activities. Int J Med Educ. 2017;8:285-9.

18. Campos J, Carlotto M, Marôco J. Copenhagen Burnout Inventory student version: adaptation and transcultural validation for Portugal and Brazil. Psicol Reflexão Crítica. 2013;26:87-97.

19. Maroco J, Campos J. Defining the student burnout construct: a structural analysis from three burnout inventories. Psychol Rep. 2012;111:814-30.

20. National Academy of Medicine. Validated instruments to assess workrelated dimensions of well-being. [consultado 2019 jan 24]. Disponível em: $\quad$ https://nam.edu/valid-reliable-survey-instruments-measureburnout-well-work-related-dimensions/\#copenhagen.

21. Grau-Alberola E, Gil-Monte P, García-Juesas J, Figueiredo-Ferraz H. Incidence of burnout in Spanish nursing professionals: a longitudinal study. Int J Nurs Stud. 2010;47:1013-20.

22. Vicente $\mathrm{C}$, Oliveira R, Maroco J. Análise fatorial do inventário de burnout de Maslach (Mbi-Hss) em profissionais portugueses. Psicol Saúde Doenças. 2013;14:152-67.

23. Cecil J, McHale C, Hart J, Laidlaw A. Behaviour and burnout in medical students. Med Educ Online. 2014;19:25209.

24. Gerber M, Brand S, Elliot C, Holsboer-Trachsler E, Pühse U, Beck J. Aerobic exercise training and burnout: a pilot study with male participants suffering from burnout. BMC Res Notes. 2013;6:78.

25. Slade A, Kies $S$. The relationship between academic performance and recreation use among first-year medical students. Med Educ Online. 2015;20:1-8.

26. Loureiro E, Mcintyre T, Mota-Cardoso R, Ferreira M. Estudo da relação entre o stress e os estilos de Psicologia, vida nas estudantes de Medicina. Acta Med Port. 2008;21:209-14.

27. Fredricks J, Eccles J. Extracurricular involvement and adolescent adjustment: Impact of duration, number of activities, and breadth of participation. Appl Dev Sci. 2006;10:132-46. 